\title{
SPOTTY APPEARANCE OF THE SOLAR DISK AS INFERRED FROM THE COMPARAISON BETWEEN EUV AND RADIO INTENSITIES
}

\author{
by Z. Sutmoto and F. Morryama \\ (Tokyo Astronomical Observatory,Tokyo, Japan)
}

RÉsumt. - On compare l'intensité du rayonnement $U V$ du Soleil avec celle que l'on peut calculer à partir d'un modèle dérivé des observations radioélectriques en précisant un travail présenté au COSPAR en 1962. La composante fondamentale du rayonnement radioélectrique entre 1000 et $9400 \mathrm{MHz}$ données par $\mathrm{H}$. TANAKA est utilisée pour établir une relation entre la température électronique et le nombre total d'électrons lors des tirs de 1960 et 1961. Les résultats justifient notre précédente hypothèse : les intensités radioélectriques auxquelles l'intensité des raies $U V$ de la couche de transition doit être comparée sont en première approximation très proches de celles du minimum d'activité solaire.

D'autre part, le nombre total d'électrons déduit de l'intensité des raies $U V$ a été obtenu à partir des données qui ont été employées par PoTTasOH, ce qui élimine quelque arbitraire dans le choix de données encore incertaines.

L'accord est encore loin d'être obtenu, les raies UV étant plus de 10 fois plus intenses que ce que le modèle permet de prévoir.

Si l'on passe à un modele inhomogène (disque solaire brillant par taches occupant $15 \%$ de la surface visible) on peut éliminer ce désaccord.

ABSTRACT. - This is a revised version of the work reported to the COSPAR Symposium of 1962 . In the first place, the basic components of the radio intensities ranging from $1000 \mathrm{MHz}$ to $9400 \mathrm{MHz}$ derived by $\mathrm{H}$. TANAKA were used to establish the relation between the electron temperature and integrated number of electrons for 1960 and 1961 fights. The result justifies our former assumption that the radio intensities with which the $E U V$ line intensities from the transition layer are to be compared should, as a first approximation, be very close to those at sunspot minimum.

In the second place, the integrated numbers of electrons from the $E U V$ line intensities were derived based on the same data as were used by POTTASCH. This is to eliminate any arbitrariness in adopting parameters which are still uncertain.

The result is that we still have a large amount of disagreement between the two sets of intensities in the sense that $E U V$ line intensities are at least ten times stronger as might be inferred from the radio intensities. If one assumes an inhomogeneous model in which the solar disk is bright only in patches covering about 0.15 of its total area, the discrepancy would be eliminated.

Резюме. - Сравнена интенсивность излучения UV Солнца с интенсивностью, которую можно вычислить исходя из модели выведенной из радио электрических наблюдений уточнив работу представленную в COSPAR в 1962 г. Основная составляющая радиоэлектрического излучения между 1000 и 9400 Мгц приведенная Танака была использована для установления соотношения между электронной температурой и общим числом электронов во время запусков 1960 и $1961 \mathrm{r}$. Результаты оправдывают нашу предшествующую гипотезу : радиоэлектрические интенсивности, с которыми должна быть сравнена интенсивность линии UV граничного слоя, являются, в первом приближении, очень близки к интенсивностям в минимум солнечной активности.

C другой стороны, общее число электронов, выведенное из интенсивности линий UV, было получено исходя из данных, которые были употреблены Роттаsсн, что устраняет некоторую произвольность в выборе еще недостоверных данных.

Согласие еще далеко не получено, линии UV будучи более интенсивными больше чем в 10 раз, чем то что позволяет предвидеть модель.

Это разногласие может быть устранено, если перейти к неоднородной модели (блеск солнечного диска пятнами, занимающими $15 \%$ видимой поверхности). 


\section{INTRODUCTION.}

In the work [7] reported to the COSPAR Symposium in Washington in 1962, we compared the EUV radiations emerging from the transition layer with the radio intensities of the quiet Sun at sunspot minimum, and found a big discrepancy between the two sets of observations. We suggested, therefore, that the discrepancy could be eliminated if we introduce a non-unifom model in which the transition layer covers the solar disk only partially, presumably, in patches.

The reason for adopting radio intensities of the quiet Sun at sunspot minimum, instead of adopting those on the day of the flight was as follows. There is no evidence that the transition layer component of the radio intensities changes remarkably according to the solar activity. Large changes of radio intensities over a wide range of frequencies with the solar activity could be interpreted as being due to the localized active regions like coronal condensations plus small change in electron density in the general corona. In fact, if big changes in radio intensities were primarily due to the changes in the transition layer, the EUV line of medium excitation would have changed remarkably from 1957 or 1960 to 1961 flight, which was not the case. It is to be mentioned that even the most recent observation of HiNTEREgGrer made on the quiet Sun on December 12, 1963 [3] also gives about the same intensities as the former flights for medium excitation lines. This would justify our procedure adopted in 1962.

Prior to and after our work, a number of works have been reported dealing with the same kind of comparison between radio and EUV [4], [5], [6]. In most cases EUV intensities were compared with the radio intensities on the day of the flight. This procedure looks to be very reasonable at first sight. However, this would only be justified, if we knew a complete set of relative models concerning the transition layer, coronal condensation and general corona, for the quiet part as well as for the active part of the Sun, on the day of the flight. Unless we knew how to separate all of those various components, the comparison of this kind would not yield anything convincing concerning any single component involved.

What we have been doing was, therefore, to concentrate to the transition layer of the quiet Sun for which, we believe, reasonably good approximation could be applied. In what follows fur- ther justification for this approximation will be given based on the radio data recently made available.

Going over to the EUV lines, we would like to emphasize that the high excitation lines such as $\mathrm{Mg}$ and $\mathrm{Fe}$ constitute a separate problem than the medium excitation lines. Because the former would certainly originate in strongly localized active parts of the Sun like coronal condensations, and are consequently strongly time dependent, whereas the latter do not change appreciably even during the sunspot cycle and primarily representative of the transition layer of the quiet part of the Sun which occupies, presumably, much larger area than plages for most of the time.

In the transition layer we would be pretty certain that, for example, some ion of $O$ is co-existent with some ion of $\mathrm{N}$ more or less in the same temperature range, and one could talk about the relative abundance of these elements. The circumstance here would be, in a sense, more favourable than in the photosphere where one would need a good model relating the temperature and the electron pressure with the optical depth. However, this advantage remains valid only when we are certain that the lines to be compared originate from the same volume element. In the case of strongly localized lines like those of $\mathrm{Mg}$ and $\mathrm{Fe}$, etc..., one would have to be very careful in talking about their relative abundances, unless the overlapping of the temperature ranges from which individual lines originate are ample enough to fulfill the above requirement.

In this paper we tried to use the same data as PotTasch [6] tabulated, with only slight modifications, in order to be as free as possible from the uncertainties in parameters we have to use, i.e., observed intensities, cross sections for ionizations and excitations, etc. But the essential difference lies in the fact that we excluded high excitation ions and thus concentrated to the transition layer only.

\section{Radio InTENSITIEe}

As a basic assumption let us assume that the plage regions do not contribute so much to the intensities of the medium excitation EUV lines as to affect their magnitude considerably. The assumption has been proved to be very close to reality by the fact that these lines have not changed appreciably from 1957 or 1960 to 1963 . On this assumption one can exclude the slowly varying component of radio. What is left in radio is then 
the quiet Sun component which still shows a slow variation over sunspot cycle by some hitherto unknown amount, due either to the general change in the corona or to the general change in the transition layer. Very recently $H$. 'TANAKa [8] studied this change in what is called the " quiet Sun component ", which he prefers to call " basic component" allowing some amount of time variation, during an entire solar cycle at four frequencies; i.e., $1000,2000,3750$ and $9400 \mathrm{MHz}$.

If one looks at the spectrum of the increment of the flux of the basic component from the sunspot minimum, one would notice that the spectrum is very flat both in 1960 and in 1961, as shown on Figure 1. This would indicate, as will be

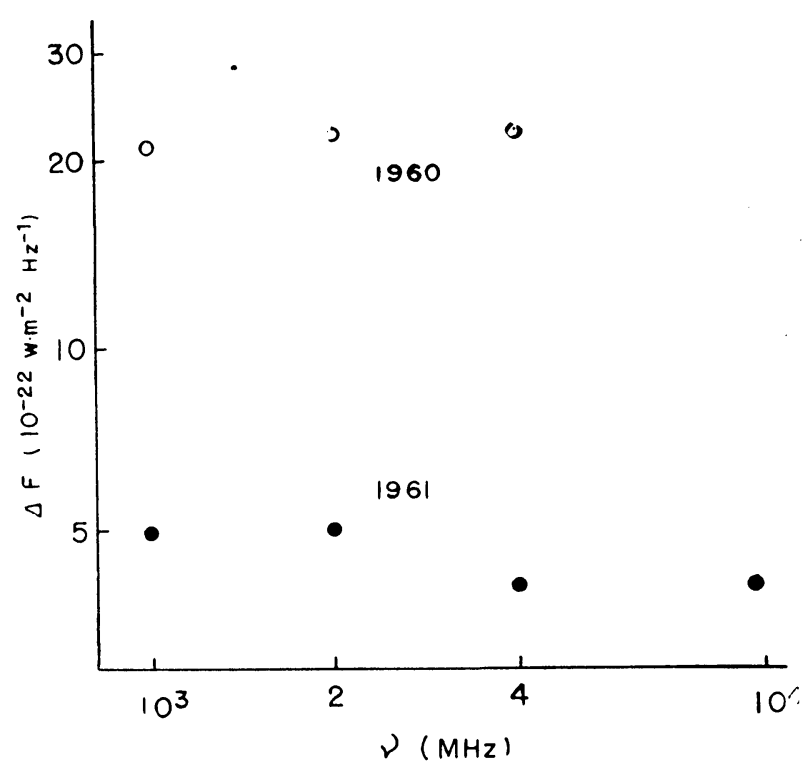

Fig. 1. - Spectrum of the increment $\Delta F(\nu)$ of the basic cumponent from the sunspot minimum for 1960 and 1961. Note that $\Delta F(v)$ stays constant against frequency in both cases.

shown in what follows. that the atmosphere to be added to the sunspot minimum solar model is to be optically thin in order to give the right amcunt of increment at all frequencies.

The radio flux at a certain frequency is given by

$$
\mathrm{F}(v)=\frac{2 k v^{2}}{c^{2}} \int \mathrm{T}_{b} d \Omega,
$$

where $T_{b}$ means the brightness temperature, which in turn is given, for thermal radiations, by

$$
\mathrm{T}_{b}=\int \mathrm{T}_{e} e^{-\tau} d \tau \text {. }
$$

The optical depth is given by

$$
\tau=\int x d h,
$$

where

$$
\varkappa=\frac{\zeta}{\nu^{2}} \frac{\mathrm{N}_{e}^{2}}{\mathrm{~T}_{e}^{3 / 2}}
$$

If we introduce a new variable $z$ defined by

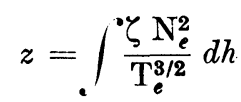

it follows that

$$
\mathrm{T}_{b}(v)=\frac{1}{v^{2}} \int \mathrm{T}_{e}(z) e^{-\frac{z}{v^{2}}} d z,
$$

and hence

$$
\mathbf{F}(v)=\frac{2 k}{c^{2}} \iint_{e}^{0} \mathrm{~T}_{e}(z) e^{-\frac{z}{v^{2}}} d z d \Omega .
$$

If we distinguish quantities relating to sunspot minimum and to other phases of sunspot cycle by putting suffixes 0 and 1 , respectively, the increment could be written as

$$
\begin{aligned}
\Delta \mathbf{F}(v) \equiv \mathrm{F}_{1}(v) & -\mathrm{F}_{0}(v) \\
& =\frac{2 k}{c^{2}} \int \dddot{\rho}\left\{\mathrm{T}_{1}(z)-\mathrm{T}_{0}(z)\right\} e^{-\frac{z}{v^{2}}} d z d \Omega .
\end{aligned}
$$

As a reasonable assumption, we assume that at any given $z, \mathrm{~T}_{1}(z)>\mathrm{T}_{0}(z)$. Because of the exponential factor in the integrand, the flux increment has in general to be always larger at higher frequencies. Only in the case

$$
\tau \equiv \frac{z}{\nu^{2}} \ll 1,
$$

i.e., in the case in which the temperature increase occurs in those regions of the solar atmosphere where the optical depth is sufficiently small at all frequencies we are concerned, can the spectrum of the increment of the flux be flat as is observed. This result would locate the atmosphere, to be added to the sunspot minimum Sun, in the corona without introducing any change in the transition layer.

Most probable curves to be compared with the EUV lines are drawn in Figure 2 for the two flights of 1960 and 1961, together with the sunspot minimum curve. One would notice that the difference from the sunspot minimum curve is very small in the transition layer.

Of course we would have to admit that the uncertainties in $\Delta \mathbf{F}(v)$ values are relatively large and it might be that $\Delta F(\nu)$ does vary with the frequency. The highest possible estimate of these uncertainties in $\Delta F(v)$ in 1960 would be

$$
10 \times 10^{-22} \mathrm{wm}^{-2} \mathrm{H}^{-1} \text {. }
$$


So assuming that in $1960, \Delta \mathrm{F}\left(9400^{\top} \mathrm{MHz}\right)$, was larger than $\Delta \mathrm{F}(1000 \mathrm{MHz})$ by this amount, one would be able to estimate the possible change of $\mathrm{T}_{e}$ in much lower regions.

By integrating the last equation by $\Omega$, one gets

$$
\Delta \mathrm{F}(v)=\frac{4 k}{c^{2}} \int \Delta \mathrm{T}_{e}(z) \mathrm{E}_{2}\left(\frac{z}{v^{2}}\right) d z \Omega_{\odot},
$$

where

$$
\mathrm{E}_{n}(x)=\int_{1}^{\infty} \frac{e^{-\infty}}{y n} d y
$$

and $\Omega_{\odot}$ means the angular area of the Sun.

Introducing numerical values, we get

$$
\Delta \mathrm{F}(v)=3.7 \times 10^{-44} \int \Delta \mathrm{T}_{e}(z) \mathrm{E}_{2}\left(\frac{z}{v^{2}}\right) d z .
$$

For simplicity we put

$$
\Delta \mathrm{T}_{\mathrm{c}}(z)=\mathrm{const}=\Delta \mathrm{T}_{\mathrm{c}} \text { for } \quad o \leqslant z \leqslant z_{c},
$$

and

$$
\Delta \mathrm{T}_{e}(z)=0 \text { for } z>z_{e}
$$

then

$$
\Delta \mathrm{F}(v)=3.7 \times 10^{-44} \Delta \mathrm{T}_{6} v^{2} \mathrm{P}\left(\frac{z}{v^{2}}\right)
$$

where

$$
\mathrm{P}(x)=\frac{1}{2}-\mathrm{E}_{\mathrm{g}}(x)
$$

For

we get

$$
z_{\mathrm{c}}=10^{10},
$$

$$
\Delta \mathrm{T}_{e}=3.5 \times 10^{3} \text {, }
$$

which is shown in Figure 2 by a dotted curve. For still smaller values of $z_{c}$ we get very unnatural curves with a big discontinuity which is not apparent on the trend of EUV lines, and these were entirely disregarded.

However, in view of the circumstance that the spectrum $\Delta \mathbf{F}(v)$ shows consistently flat character from year to year, the possibility of the dotted curve being real is very small. Nevertheless, it might be remarked that even the improbable change such as this in lower atmospheres would not make any big difference from the sunspot minimum curve.

\section{Extreme Ultraviolet tinkes.}

In the former work we made use of the WoolLEY and AlleN's formulas [9] for the ionization and excitation of the EUV lines, and for the excitation cross sections we used ALLEN's rule of $f$ values [1]. These points have been revised by POTTASOH in his discussion of the chemical composition in the lower corona ${ }^{\top}[6]$. The essential difference is that $f$ values we used before were on the average about three times larger than the new values. In order to make the comparison between the two works easier we adopted the values of

$$
\frac{\mathrm{N} \text { (element) }}{\mathrm{N}(\mathrm{H})} \int_{\mathbf{R}} \mathrm{N}_{e}^{2} d h,
$$

tabulated by PotTasoH in column (9) of Table II.

In order to convert these values into $z_{R}$ values, they were first divided by $\mathrm{T}_{\max }^{3 / 2}$, and then the chemical abundance of GOLDBERG, MüLLER and Aller [2] was used. The suffix $R$ in $z$ denotes that the integration over the height was effected only in the temperature range in which the line in question is bright. In order to compare with the radio curre in Figure 2, we have to extend

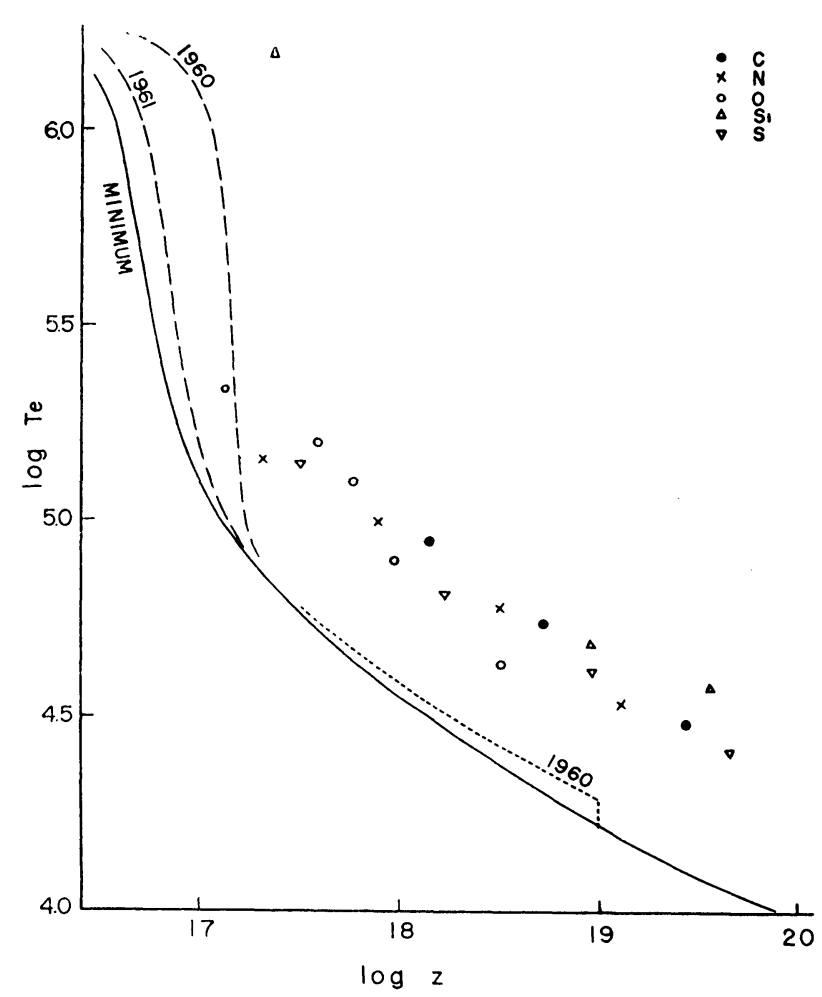

Fra. 2. - Empirical $\log \mathrm{T}_{e}$ versus $\log z$ relations for both radio and EUV. For the radio, continuous curve represents the quiet Sun at sunspot minimum, broken curves indicate most probable radio curves with which the EUV lines are to be compared for the years 1960 and 1961, respectively and the dotted curve shows a kind of curve when large errors are assumed in the spectrum of Figure 1 for 1960.

the integration to infinity starting from some level, where the temperature is of certain value $T_{\max }$. This integration was made simply by adding the $z_{\mathrm{R}}$ 's of still higher ionization stages of the element under consideration with the precaution that at 
the lowest temperature range corresponding to a $\mathrm{T}_{\max }$ only one half of $z_{\mathrm{R}}$ was added. In this way one could get a set of $z$ values for a number of $\mathrm{T}_{\max }$ 's for each element. However, this procedure would give $z$ values which were not integrated to infinity but integrated only up to the highest ionization stage available for that particular element. Beyond this limit we actually have no data. For example, in the case of 0 , one can get the right values of $z$ starting from $O$ VI to lower ionization stages, except for a constant term, which represents the contribution of still higher layers.

One cannot make use of the values inferred from $\mathrm{Ne}, \mathrm{Mg}$ and $\mathrm{Fe}$, which undoubtedly come from regions with still higher temperatures, as was done by PотTasCH because these high excitation lines are emitted from strongly localized regions of the corona and do not represent anything of the quiet corona above the normal transition layer with which we are dealing. The best we can do would be to plot the $z$ values calculated in the way described above on Figure 2, admitting that the actual $z$ values might be a little larger by some unknown amount.

The amount to be added is the same for a single element, but varies from element to element, depending upon the highest temperature available to that element. The apparent good coincidence between the different elements plotted in Figure 2 is, therefore, only apparent. Among those elements like $\mathrm{C}, \mathrm{N}, \mathrm{O}, \mathrm{Si}$, and $\mathrm{S}$ there are actually some discrepancies by a factor of about 2, just as was pointed out by PоттAsCH. From the reasons described in Section 1, we are not in a position to discuss the abundances of $\mathrm{Ne}, \mathrm{Mg}$, and Fe. We would like to mention, however, that the overlapping of the temperature ranges among those elements does not look ample enough at the present, when one takes into account the relatively large uncertainties both in observation and in formulation.

\section{Discussions.}

What strikes one most in looking at Figure 2 would be the large discrepancy between the radio curve and the average trend of the EUV points. The discrepancies among non-hydrogen elements by a factor of 2 could more easily be attributed to uncertainties of some kind. It shoud also be noted that the discrepancy by a factor of 10 is a lower limit. There are three reasons which might make the discrepancy even larger :(1) $z$ values for EUV points are still to be increased by some amount representing the normal corona. (2) If dielectronic recombination is effective in this case also, it would make the temperature scale for the EUV points higher without affecting the radio curve. (3) Correction for self-absorption, if important, makes the actual $z$ values for EUV points larger.

The discrepancy between the radio and the EUV lines could be described in terms of the abundance anomaly in hydrogen to non-hydrogen ratio. Then the question arises, why we found again the hydrogen deficiency, whereas PotTascH did not find any anomaly in $\mathrm{H} / \mathrm{O}$ ratio ?

Basic difference between PotTasch's work and ours is that PoTTASCH compared the EUV lines of all excitations with the radio intensity of sunspot maximum, whereas we compared the EUV lines of medium excitations with the radio intensity of sunspot minimum, or more precisely with the basic components in the years of the flights. This situation makes the discrepancy between radio and EUV less in his case than in our case. However, if Potrasch is right, the $\mathrm{O}$ lines would have decreased by a factor of 10 from the earliest flights to the most recent one. As this is not the case, we are convinced that there is a large discrepancy between radio and EUV.

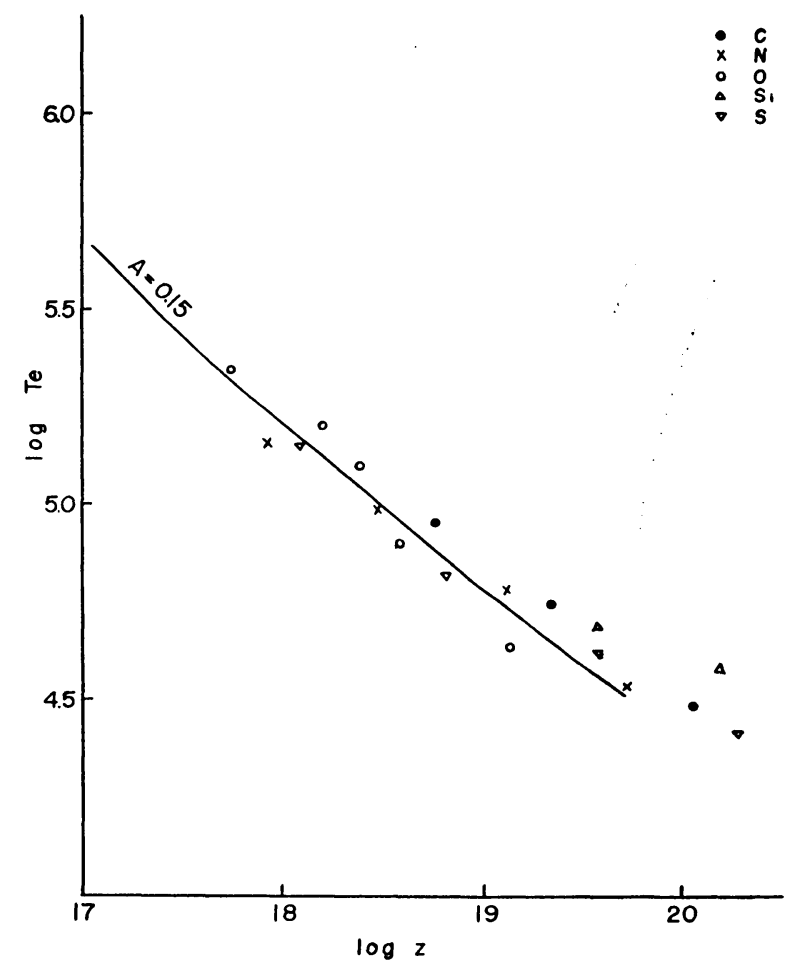

Fig. 3. - $\log T_{e}$ versus $\log z$ relation for the bright part of the solar disk when the bright part covers only $15 \%$ of the disk. 
One way to elucidate this discrepancy is simply to attribute it to a hydrogen deficiency by a factor of about 10 in the corona as compared with the photosphere. But it does not seem very likely.

Another way of interpreting this is in terms of inhomogeneity, as we have been suggesting. If the solar disk is only partly bright in both radio and medium excitation EUV lines, the actual brightness of the bright part would be brighter than the average, inversely proportionally to the fraction of the bright area A over the disk. This would mean in the case of radio, that at $\tau \sim 1$, i.e., at a fixed value of $z$, the brightness temperature, accordingly the electron temperature is higher by this factor of $1 / \mathrm{A}$. Of course in this process we will have to take care of the background chromospheric radiation of about $6000^{\circ}$ based on the equation,

$$
\mathrm{T}_{e}(z)=\mathrm{A} \mathrm{T}_{1}(z)+(1-\mathrm{A}) \mathrm{T}_{2}(z),
$$

where $T_{e}$ the average temperature, $T_{1}$ the temperature in the bright part, and $T_{2}$ is the background temperature of $6000^{\circ}$. On the other hand, in the case of EUV lines, this implies an increased number of active ions along the line of sight, i.e., an increase in $z$ in a fixed temperature range, by the same factor of $1 / \mathrm{A}$. Summing up, by this transformation the radio curve goes up, whereas the EUV points move to the right in Figure 2.

By adopting a suitable value of $A$ one would be able to bring the two sets of observations into coincidence. In our present case $A=0.15$ gives the coincidence as is seen in Figure 3. However, numerical values of A are still very preliminary, as there are reasons to believe that the value might even be smaller. The significant point of our conclusion is that it is very likely that the normal transition layer does not cover the whole solar disk uniformly but covers only in patches or in spots, presumably, associated with the spicule structure of the chromosphere.

In conclusion we would like to emphasize the importance of carrying out as many absolute intensity measurements of EUV lines as possible during this sunspot minimum. This would help towards investigating the active Sun following up the minimum.

Manuscrit reçu le 30 septembre 1964.

\section{REFERENCES}

[1] Allen C. W., 1960, 8 th Liège Symposium, 241.

[2] Goldberg L., Müller E. A. and Aller L. H., 1960, Ap. J. Suppl., 5, No 45.

[3] Hinteregger H. E., Private communication.

[4] Ivanov-Kholodny G. S. and Nmolsky G. M., 1961, A. J. USSR, 38, 45.

[5] Koyama S., 1963, Publ. Astr. Soc. Japan, 15, 15.

[6] Рottasch S. R., 1963, Ap. J., 137, 945.
[7] Suemoto Z. and Moriyama F., 1963, Space Research, III, W. Priester, Editor. North Holland Publ. Co., $1963,800$.

[8] Tanaka H., 1964, Proc. Res. Inst. Atmospherics, Nagoya Univ., 11, 41.

[9] Woolley R. v. d. R. and Allen C. W., 1950, $M . N ., 110,358$.

\section{Discussion}

W. A. Fowler. - Would a reduction of the heavy element abundances by a factor 10 remore the discrepancy discussed by Dr. Sümoto ?

Z. Suemoto. - Yes. 